\title{
Energy-Aware Flexible Job Shop Scheduling Using Mixed Integer Programming and Constraint Programming
}

\author{
Andy Ham $\mathbb{D}^{1},{ }^{1}$ Myoung-Ju Park $\mathbb{D}^{2},{ }^{2}$ and Kyung Min Kim $\mathbb{D}^{3}$ \\ ${ }^{1}$ Applied Engineering Technology, North Carolina A\&T State University, Greensboro, NC 27411, USA \\ ${ }^{2}$ Industrial and Management Systems Engineering, Kyung Hee University, Seoul, Republic of Korea \\ ${ }^{3}$ Industrial Management and Engineering, Myong Ji University, Seoul, Republic of Korea \\ Correspondence should be addressed to Myoung-Ju Park; pmj0684@khu.ac.kr
}

Received 7 April 2021; Revised 16 June 2021; Accepted 22 June 2021; Published 30 June 2021

Academic Editor: Snežana Tadić

Copyright (@ 2021 Andy Ham et al. This is an open access article distributed under the Creative Commons Attribution License, which permits unrestricted use, distribution, and reproduction in any medium, provided the original work is properly cited.

Compromising productivity in exchange for energy saving does not appeal to highly capitalized manufacturing industries. However, we might be able to maintain the same productivity while significantly reducing energy consumption. This paper addresses a flexible job shop scheduling problem with a shutdown (on/off) strategy aiming to minimize makespan and total energy consumption. First, an alternative mixed integer linear programming model is proposed. Second, a novel constraint programming is proposed. Third, practical operational scenarios are compared. Finally, we provide benchmarking instances, CPLEX codes, and genetic algorithm codes, in order to promote related research, thus expediting the adoption of energy-efficient scheduling in manufacturing facilities. The computational study demonstrates that (1) the proposed models significantly outperform other benchmark models and (2) we can maintain maximum productivity while significantly reducing energy consumption by $14.85 \%$ (w/o shutdown) and $15.23 \%$ (w/shutdown) on average.

\section{Introduction}

Energy consumption is a very important issue for our society in terms of both environment and economy. U.S. Energy Information Administration [1] recently reported international energy outlook 2019 with projections to 2050. In the report, the world energy consumption is expected to increase by approximately $50 \%$, accounting for more than half of the non-OECD Asian countries, including China and India. Industrial sectors, such as manufacturing, agriculture, and construction, are the largest consumer among end users, and their energy consumption will increase by more than $30 \%$ from 2018 to 2050 . The share of energy consumed by energyintensive manufacturing holds steady at 50\% from 2018 to 2050. These predictions and phenomena were analyzed in several studies [2-4].

Manufacturing has enormous potential for energy saving because $80 \%$ of the energy consumed by machines occurs in the idle state [5]. For this reason, several methods to increase energy efficiency in manufacturing have been attempted.
The common method is to optimize a production schedule by considering green metrics along with traditional performance indicators. This approach called energy-aware scheduling or energy-efficient scheduling has the advantage of achieving significant performance at no extra cost [6].

In this paper, we deal with energy-efficient scheduling in the flexible job shop scheduling problem (FJSP). Mouzon et al. [5] observed that if an idle period is long enough, the energy could be saved by using shutdown (on/off) strategy to turn the machine off and on. Che et al. [7] first developed a mixed integer linear programming (MILP) model for a single machine scheduling with a shutdown strategy, and the model was effectively validated with CPLEX solver. Subsequently, the shutdown strategy was applied to various production systems, and the first MILP model for FJSP with a shutdown strategy was proposed by Zhang et al. [8]. However, the proposed model was complex and computationally inefficient, so Meng et al. [9] improved it. However, both studies used only one type of MILP formulations for FJSP. This paper proposes the most efficient MILP formulation. 
The contributions of this paper are threefold. First, we propose an alternative MILP model for FJSP with a shutdown strategy. Second, we devise a constraint programming (CP). Third, the experimental study demonstrates that we can reduce energy consumption by $14.85 \%$ (w/o shutdown) and $15.23 \%$ (w/shutdown) on average, while not compromising productivity. In addition, we provide benchmarking instances, CPLEX MIP and CP source codes, and GA code, in order to promote related-research, thus expediting the adoption of energy-efficient scheduling in manufacturing facilities.

The remainder of the paper is organized as follows: Section 2 shows the literature review of FJSP, energy-efficient scheduling, and constraint programming. In Section 3, the problem description and solution methods are described. In Section 4, we compare the performance of proposed models and existing model through computational experiments. Finally, the conclusion and some directions for future research are given in Section 5.

\section{Literature Review}

2.1. Flexible Job Shop Scheduling Problem. The FJSP has been extensively studied over the past 30 years. A variety of techniques from exact methods to heuristics have been used in this research. Exact methods include branch-and-bound algorithm, MILP, and Lagrangian relaxation method among others, while heuristics include ant colony optimization, artificial bee colony, artificial immune system, evolutionary algorithms, greedy randomized adaptive search procedure, neighborhood search, particle swarm optimization, simulated annealing, Tabu search, hybrid techniques, and so on. See Chaudhry and Khan [10] for the survey about FJSP. We focus on the MILP method.

MILP models for FJSP are classified into four different types by the main binary decision variable. The first is the machine-position formulation (MPF) that determines the position of the machine, where each operation is processed $[8,9,11,12]$. The second is the general precedence formulation (GPF) that determines whether one operation precedes the other operation on the same machine $[13,14]$. Note that one operation is not necessarily positioned immediately before the other operation. The third is the immediate precedence formulation (IPF) that determines whether one operation immediately precedes the other operation on the same machine [15]. The fourth is the time indexed formulation (TIF) that determines the time of the machine when each operation is started [16]. Demir and Kürşat Isleyen [17] classified mathematical models by this criterion and compared the computational results of MILP models.

2.2. Energy-Efficient Scheduling. The studies for energy-efficient scheduling are classified into four groups based on saving methods. The first is to reduce unnecessary idle time. This is a model that extends the existing model to save energy by reducing unnecessary idle time. The second is to shut down the idle machines. Turning off machines during the idle can save energy when idle time is long enough. The decision when to shut down a machine is added into the traditional scheduling model. The third is to slow down a machine speed. Energy consumption depends on a speed of a machine. We can save energy by adjusting a speed of a machine without impacting the makespan. The fourth is offpeak production. In the peak time, electricity costs are high. Therefore, a production at nonpeak time can save a significant amount of electricity costs. Table 1 categorizes studies on energy-efficient scheduling by this criterion. See Gahm et al. [37] and Gao et al. [38] for reviews of energyefficient scheduling.

Our study belongs to the energy-efficient FJSP using the shutdown option. Among the studies, Dai et al. [25] and $\mathrm{Wu}$ and Sun [26] developed a genetic simulated annealing algorithm. Zhang et al. [8] developed MILP model and discovered energy-efficient rules that could be implemented in real practice. Meng et al. $[9,27]$ proposed several effective MILP models and evaluated the performance of these models. Zhang et al. [8] and Meng et al. [9, 27] used MPF, while our paper uses IPF. Moreover, we develop constraint programming and genetic algorithm models.

2.3. Constraint Programming. Hiller and Lieberman [39] noted that no presentation of the basic ideas of MILP is complete these days without introducing $\mathrm{CP}--$ that is promising to greatly expand our ability to formulate and solve various scheduling problems. CP has been applied to various scheduling problems, demonstrating a rapid computational speed. The search within IBM CPLEX CP Optimizer is equipped with the presolve functionality, some constraint propagation algorithms, temporal linear relaxation used to guide the search, and two search space exploration strategies that are used concurrently: the large neighborhood search for producing good quality solutions and failure-directed search for proving infeasibility or optimality [40].

\section{Problem Description and Solutions}

Consider a flexible job shop environment that consists of a set of heterogeneous machines $(k \in K)$ and a set of jobs $(i \in I)$. Each job $i$ consists of a set of operations $\left(J_{i}=\left\{1, \ldots, n_{i}\right\}\right)$. Each operation needs to be processed in a specific order (known as precedence constraints) for a given job. A machine can perform at most one operation at a time. Each operation must be processed by one of qualified machines. In addition to the standard FJSP problem, an amount of energy consumption is considered in this energyaware scheduling approach. The energy is consumed during production, idle, and shutdown. The production requires the highest amount of energy, while the idle does the medium and the shutdown requires the lowest. In particular, the shutdown can be enforced when a continuous idle period of a machine is expected to be long enough to compensate the shutdown penalty. When idle times are inevitable, the key is to adjust small-size idle intervals and locate them in a single large-size interval to turn on a long-size shutdown. 
TABLE 1: Articles including energy-efficient scheduling models.

\begin{tabular}{|c|c|c|}
\hline Saving method & Environment & References \\
\hline \multirow{4}{*}{ Reduce idle time } & Single machine & Jiang et al. [18] \\
\hline & Hybrid flow shop & Li et al. [19]; Zhang et al. [20] \\
\hline & JSP & Jiang et al. [21] \\
\hline & FJSP & Yin et al. [22]; Jiang et al. [23] \\
\hline \multirow{5}{*}{ Shutdown idle machines } & Single machine & Mouzon et al. [5]; Che et al. [7] \\
\hline & Hybrid flow shop & Meng et al. [24] \\
\hline & Meta Heuristic & Dai et al. [25]; Wu and Sun [26] \\
\hline & MILP-MPF & Zhang et al. [8]; Meng et al. [9]; Meng et al. [27] \\
\hline & MILP-IPF & This study \\
\hline \multirow{4}{*}{ Exploit variable machine speed } & Single machine & Che et al. [28] \\
\hline & Flow shop & Fang et al. [29]; Mansouri et al. [30] \\
\hline & JSP & Zhang and Chiong [31] \\
\hline & FJSP & Zhang et al. [32] \\
\hline \multirow{3}{*}{ Shift production soft-enter to off-peak } & Single/parallel machine & Rager et al. [33] \\
\hline & Hybrid flow shop & Schulz et al. [34] \\
\hline & FJSP & Moon and Park [35]; Gong et al. [36] \\
\hline
\end{tabular}

3.1. Mixed Integer Linear Programing Models. Meng et al. [9] presented six MILP models for our problem and showed that the second model outperforms all the other models through the numerical experiments. We term this efficient model MILP-2. Despite the superiority of MILP-2, we found that it can be further improved during the implementation by employing tuples instead of arrays, thus dramatically reducing the number of binary decision variables. For instance, the MILP-2 uses a binary variable $Y_{i, j, k, t}$ that represents whether the $j$-th operation of job $i$ is processed at the $t$-th position of machine $k$. This variable is a sparse array, in which most of the elements are zero. Moreover, Meng et al. [9] set the maximum number of the positions of machine $k$ (denoted by $m_{k}$ ) to the total number of operations $\left(\sum_{i \in I} n_{i}\right)$, which is excessively large. We set $m_{k}$ to the number of operations that machine $k$ can process in the instance. By taking those measures, we were able to reduce the number of binary variables by $50-60 \%$ in the same instances. We term this efficient model MILP-2A.

MILP-2 is an extension of MPF for FJSP so that energy consumption can be considered. However, Demir and Kürşat Isleyen [17] showed that MPF is the slowest model among alternative models. Choi and Choi [15] proposed a new immediate precedence formulation (IPF) that decides whether one operation immediately precedes the other as shown in Figure 1.

Now, we will propose a new model that improves IPF and extends it to account for energy consumption. We mostly use the same notations as Meng et al. [9]. The details are as follows.

Parameters:

I: set of jobs.

$n_{i}$ : number of operations of job $i \in I$.

$J_{i}$ : set of operations of job $i \in I$, that is, $J_{i}=\left\{1, \ldots, n_{i}\right\}$.

$O$ : set of job and operation pairs, that is, $O=\left\{(i, j) \mid i \in I, j \in J_{i}\right\}$.

$K$ : set of all machines.

$K_{o}$ : set of machines which can process operation $o \in O$.

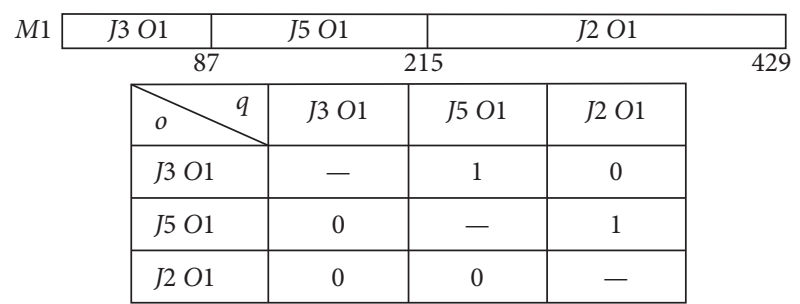

Figure 1: An illustration of immediate precedence formulation using a decision variable $W_{o, q}$.

$p_{o, k}$ : processing time of $o \in O$ by machine $k \in K_{o}$. $D_{k}$ : the unit energy consumed at the idle time for machine $k$ when the shutdown strategy is not used $P_{0}$ : the unit energy consumed by a facility for lighting, heating, and cooling during the makespan.

$E_{o, k}$ : the energy consumed when operation $o$ is processed by machine $k$.

$N_{k}$ : the maximum times of shutdown strategy for machine $k$.

$G_{k}$ : the energy consumption of machine $k$ when the shutdown strategy is used.

$T B_{k}$ : the breakeven period of machine $k$ in which the same amount of energy is consumed during the idle time whether the shutdown strategy is used or not. Hence, $T B_{k}=G_{k} / D_{k}$.

$O_{q}$ : the set of operations which can be processed by the same machine with operation $q \in O$.

$P O$ : the set of precedence operation pairs in the same job operations, that is, $P O=\{(o, q) \mid o=(i, j), q$ $\left.=(i, j+1), i \in I, j=1, \ldots, n_{i}-1\right\}$.

$M_{1}=\sum_{o \in O} \max \left\{p_{o, k} \mid k \in K_{o}\right\}$.

$M_{2}=\max \left\{D_{k} \cdot T B_{k} \mid k \in K\right\}$.

Decision variables:

$X_{o, k}: 1$ if $o \in O$ is processed by machine $k \in K_{o}$ and 0 otherwise. 
$S_{o}$ : start time of $o \in O$.

$C_{o}$ : completion time of $o \in O$.

$C_{\max }$ : makespan,

that

is,

$C_{\text {max }}=\max \left\{c_{o} \mid o=\left(i, n_{i}\right) \in O\right\}$.

$V_{o}$ : idle time after operation $o \in O$.

$R_{o}$ : energy consumption during $V_{o}$.

$W_{o, q}: 1$ if $o \in O$ immediately precedes $q \in O$ on the same machine and 0 otherwise.

$Z_{o, k}: 1$ if turning shutdown strategy is implemented during $V_{o}$ on machine $k \in K_{o}$ and 0 otherwise.

$Q_{o, k}: 1$ if $o \in O$ is processed last on machine $k \in K_{o}$ and 0 otherwise.

\subsubsection{MILP-3 for Energy-Aware Flexible Job Shop Scheduling.}

$$
\begin{gathered}
\operatorname{Min} \sum_{o \in O} \sum_{k \in K_{o}} E_{o, k} X_{o, k}+\sum_{o \in O} R_{o}+P_{0} C_{\max } \\
\sum_{k \in K_{o}} X_{o, k}=1, \quad \forall o \in O, \\
C_{o}=S_{o}+\sum_{k \in K_{o}} p_{o, k} X_{o, k}, \quad \forall o \in O \\
S_{q} \geq C_{o}, \quad \forall(o, q) \in P O, \\
C_{\max } \geq C_{o}, \quad \forall o=\left(i, n_{i}\right) \in O \\
\sum_{o \in O} Q_{o, k} \leq 1, \quad \forall k \in K, \\
\sum_{o \in O_{q}} W_{o, q} \leq 1, \quad \forall q \in O, \\
\sum_{q \in O_{o}} W_{o, q}+\sum_{k \in K_{o}} Q_{o, k}=1, \quad \forall o \in O, \\
W_{o, q}-1 \leq X_{o, k}-X_{q, k}, \quad \forall o \in O, q \in O_{o}, k \in K_{o} \cap K_{q},
\end{gathered}
$$$$
X_{o, k}-X_{q, k} \leq 1-W_{o, q}, \quad \forall o \in O, q \in O_{o}, k \in K_{o} \cap K_{q} \text {, }
$$

$$
\sum_{k \in K_{o} \cap K_{q}} X_{o, k} \geq W_{o, q}, \quad \forall o \in O, q \in O_{o}
$$

$$
\sum_{k \in K_{o} \cap K_{q}} X_{q, k} \geq W_{o, q}, \quad \forall o \in O, q \in O_{o}
$$

$$
Q_{o, k} \leq X_{o, k}, \quad \forall o \in O, k \in K_{o}
$$

$$
S_{q} \geq C_{o}+M_{1}\left(W_{o, q}-1\right), \quad \forall o \in O, q \in O_{o}
$$

$$
\begin{aligned}
& \text { during } V_{o} \text {. Constraints }(22)-(23) \text { enforce that shutdown } \\
& \text { strategy can be turned during } V_{o} \text { on machine } k \text { only when } \\
& o \text { is processed by machine } k \text { and it is not the last operation } \\
& \text { of machine } k \text {. We term this newly proposed model } \\
& \text { MILP-3. }
\end{aligned}
$$

3.2. Constraint Programming. All time durations, such as makespan, shutdowns, and productions, are explicitly modeled as the interval variables as shown in Figure 2. 


\begin{tabular}{|c|c|}
\hline \multicolumn{2}{|c|}{ Makespan } \\
\hline \hline Production & Shutdown \\
\hline
\end{tabular}

Figure 2: Modeling overview.

As Ham [41] pointed out, there is no standard in CP formulation, unlike a similar MILP formulation. Therefore, this paper formulates the model using generic keywords and syntaxes as we refer to the CP formulations by Laborie et al. [40] and IBM ILOG CP Optimizer [42].

The proposed CP model is built upon the following decision variables:

$Z_{k, s}$ : interval representing $s$-th shutdown on machine $k \in K$
$C_{k}$ : interval representing makespan of $k \in K$

$T_{o}$ : interval representing operation $o \in O$

$X_{o, k}$ : interval representing operation $o \in O$ on machine $k \in K_{o}$

Seq $_{k} \leftarrow\left[X_{o, k}\right]$ : collection of variables assigned to machine $k$

$e S$ : total energy consumption during shutdowns

$e P$ : total energy consumption during productions

$e I$ : total energy consumption during idles

$e C$ : total common energy consumption

$$
\begin{aligned}
& \operatorname{Min} e P+e S+e I+e C \text {, } \\
& \text { alternative }\left(T_{o},\left[X_{o, k: k \in K_{o}}\right]\right), \quad \forall o \in O, \\
& \text { endBeforeStart }\left(T_{o}, T_{q}\right), \quad \forall(o, q) \in P O \text {, } \\
& \operatorname{noOverlap}\left(\operatorname{Seq}_{k}\right), \quad \forall k \in K, \\
& \operatorname{span}\left(C_{k},\left[X_{o, k: o \in O, k \in K}\right]\right), \quad \forall k \in K, \\
& \operatorname{startOf}\left(Z_{k, s}\right)>\operatorname{startOf}\left(C_{k}\right), \quad \forall k \in K, s \leq N_{k} \text {, } \\
& \operatorname{endOf}\left(Z_{k, s}\right)<\operatorname{endOf}\left(C_{k}\right), \quad \forall k \in K, s \leq N_{k} \text {, } \\
& \operatorname{sizeOf}\left(Z_{k, s}\right) \geq T B_{k}, \quad \forall k \in K, s \leq N_{k} \text {, } \\
& e P=\sum_{o \in O} \sum_{k \in K_{o}} E_{o, k} \operatorname{sizeOf}\left(T_{o}\right), \\
& e S=G_{k} \sum_{k \in K_{o}} \sum_{s \leq N_{k}} \text { presenceOf }\left(Z_{k, s}\right) \\
& e I=D_{k} \sum_{k \in K}\left(\operatorname{sizeOf}\left(C_{k}\right)-\sum_{s \leq N_{k}} \operatorname{sizeOf}\left(Z_{k, s}\right)-\sum_{o \in O} \operatorname{sizeOf}\left(X_{o, k}\right)\right) \\
& e C=P_{0} \underset{\forall o \in O}{\operatorname{Max}}\left\{\operatorname{endOf}\left(T_{o}\right)\right\} .
\end{aligned}
$$

Objective (24) is to minimize the total energy consumption, which is comprised of production, shutdown, idle, and common. Constraint (25) ensures that each operation is processed by exactly one machine. Constraint (26) enforces each operation starts after its precedent operation is finished. Constraint (27) prevents intervals in a sequence from overlapping. Constraint (28) determines the makespan of each machine. Constraints (29)-(30) ensure that a shutdown can occur during a makespan. Constraint (31) ensures the minimum length of each shutdown. Constraints (32)-(35) compute the total energy consumption for production, shutdown, idle, and common, respectively. We term this model CP-1.

During the preliminary study, the CP-1 could not outperform the MILP-2A model. Here, we explored an alternative CP model. In the CP-1, the idle time was computed 
based on other interval variables in Constraint (34). We here present an alternative CP model, which explicitly captures the idle intervals.

$\mathbf{I}_{k, t}$ : interval representing $t$-th idle on machine $k \in K$.

$$
\begin{aligned}
e I= & D_{k} \sum_{k \in K} \sum_{t \leq m_{k}} \operatorname{sizeOf}\left(I_{k, t}\right), \\
\operatorname{sizeOf}\left(C_{k}\right)= & \sum_{o \in O} \operatorname{sizeOf}\left(X_{o, k}\right)+\sum_{s \leq N_{k}} \operatorname{sizeOf}\left(Z_{k, s}\right) \\
& +\sum_{t \leq m_{k}} \operatorname{sizeOf}\left(I_{k, t}\right) .
\end{aligned}
$$

Constraint (36) replaces Constraint (34). Then, Constraint (37) is introduced to ensure that a makespan of the machine must be packed by productions, shutdowns, and idles. All other constraints stay in place. We term this revised model CP-2.

Figure 3 represents an optimal schedule for a small benchmark instance (mfjs07). Figure 3(a) shows the detailed production schedule per machine with an objective of makespan minimization, and Figure 3(b) depicts the schedule with an objective of energy minimization, while maintaining the same productivity. The proposed model merges the small-size idles enough to turn on shutdown strategy. For instance, the two idles that occurred in machine 4 are replaced by a single shutdown in the same machine. The energy-aware scheduling saved the energy consumption by $1.35 \%$ without hurting productivity in the mfjs 07 instance.

\section{Computational Experiments}

In this section, the effectiveness of the proposed models is examined. The MILP, CP, and flow control models are all coded in IBM OPL 12.8.0 on a personal computer with an Intel ${ }^{\circledR}$ Core i7-4770 CPU with $16 \mathrm{~GB}$ of RAM. All the test instances, MILP codes, CP codes in IBM OPL, and GA code can be found online at https:/github.com/hamcruise/FJSPShutdown.

4.1. Problem Instances. Meng et al. [9] proposed a set of test instances derived from the known benchmark instances SFJS01-10 and MFJS01-10 [12] by considering energy consumption. We adopted the same test instances. SFJS0110 are small-sized, and MFJS01-10 are medium-sized. We also adopted another FJSP benchmark test instance suggested by Behnke and Geiger [43]. Among their extensive 60 instances, we adopted the first 10 instances and added energy parameters in the same way Meng et al. [9] did. Finally, we also used another FJSP benchmark test instance suggested by Kacem et al. [44], which has been adopted by Singh and Mahapatra [45]. The detailed size of the instance $(a / b / c)$ is recorded in Table 2, where index $a$ denotes the number of jobs, $b$ denotes the maximum number of operations for a job, and $c$ denotes the number of machines.

For all instances, the common power $\left(P_{0}\right)$ and maximum times of shutdown $\left(N_{k}\right)$ are set to be 5 and 3 , respectively. The processing powers are drawn from the uniform distribution $[3,5]$. The idle power $\left(D_{k}\right)$ is randomly generated from the set $\{1,2,3\}$, and the energy consumption during shutdown $\left(G_{k}\right)$ is generated from the set $\{10,30,60\}$.

4.2. Experimental Results. Figure 4 shows the ratio of the number of binary variables of MILP models to MILP-2. As the size of MILP-2 increases, the binary variables of MILP$2 \mathrm{~A}$ and MILP-3 decrease. In particular, it is observed that the reduction ratio of the binary variable of MILP-3 sharply decreases as the size increases.

In this subsection, we implemented a genetic algorithm to compare the performance between proposed methods and metaheuristic. Genetic algorithm (GA) is known as an effective metaheuristic to solve flexible job shop scheduling problems [46-49]. The overall GA framework in Zhang et al. [50] was adopted. We had conducted a preliminary test for tuning hyperparameters with respect to the quadruple (popSize, numGen, crRate, mutRate), where popSize is the population size (popSize $\in\{100,200,400\})$, numGen is the maximum number of generations (numGen $\in\{50,100$, $200\}), c r$ Rate is the crossover rate (crRate $\in\{0.6,0.7,0.8,0.9$, $1.0\}$ ), and mutRate is the mutation rate (mutRate $\in\{0.0,0.1$, $0.2,0.3\})$. The best GA hyperparameters that were obtained from the preliminary test were as follows: popSize $=100$, numGen $=50$, crRate $=0.7$, and mutRate $=0.1$. The increasing population size and number of generations did not ensure obtaining better solutions. The value of the fitness function converged fast in practice.

Tables 3 and 4 compare the proposed MILP and GA with $\mathrm{CP}$ models in terms of the objective function value (total energy consumption) and computation times within 600 seconds for Fattahi and Behnke's instances, respectively. Column 1 identifies the name of the instance, columns 2-7 include the total energy consumption, and columns 8-13 report the computation times. The bold font indicates the optimality.

Table 3 reports the results based on Fattahi's instances. For small-sized instances (SFJS01-10), all approaches found the optimal solutions, except GA. In computation times, all approaches, except CP-1, terminated in one second. For medium-sized instances (MFJS01-10), CP-2 yielded the best results on average. In computation times, GA significantly outperforms all other approaches seconds. However, MILP2, MILP-2A, and MILP-3 terminated at a similar time.

Table 4 reports the results based on Behnke's instances. The proposed MILP-2A performed the best in average. GA is significantly faster than the MILP and CP approaches. However, the total energy consumption is increased up to $8.1 \%$ on average.

In order to determine if the means of two sets of data are significantly different from each other, we conducted the $t$ test: Paired Two-Sample for Means at an alpha level of 0.05. Table 5 indicates that, for medium-sized Fattahi's instances, there is a statistically significant difference between CP-2 vs. CP-1 and GA, while there is no statistically significant difference between CP-2 vs. MILP-2, MILP-2A, and MILP-3. For Behnke's instances, there is a statistically significant difference between MILP-2A vs. CP-1, MILP-2, MILP-2A, 


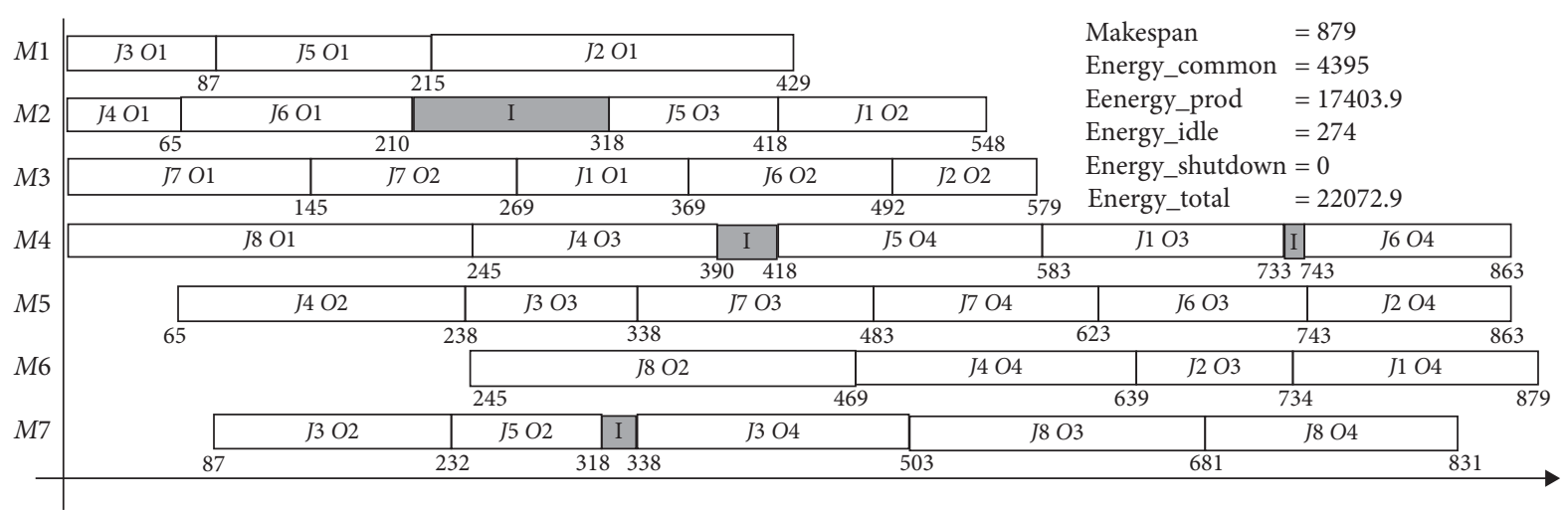

(a)

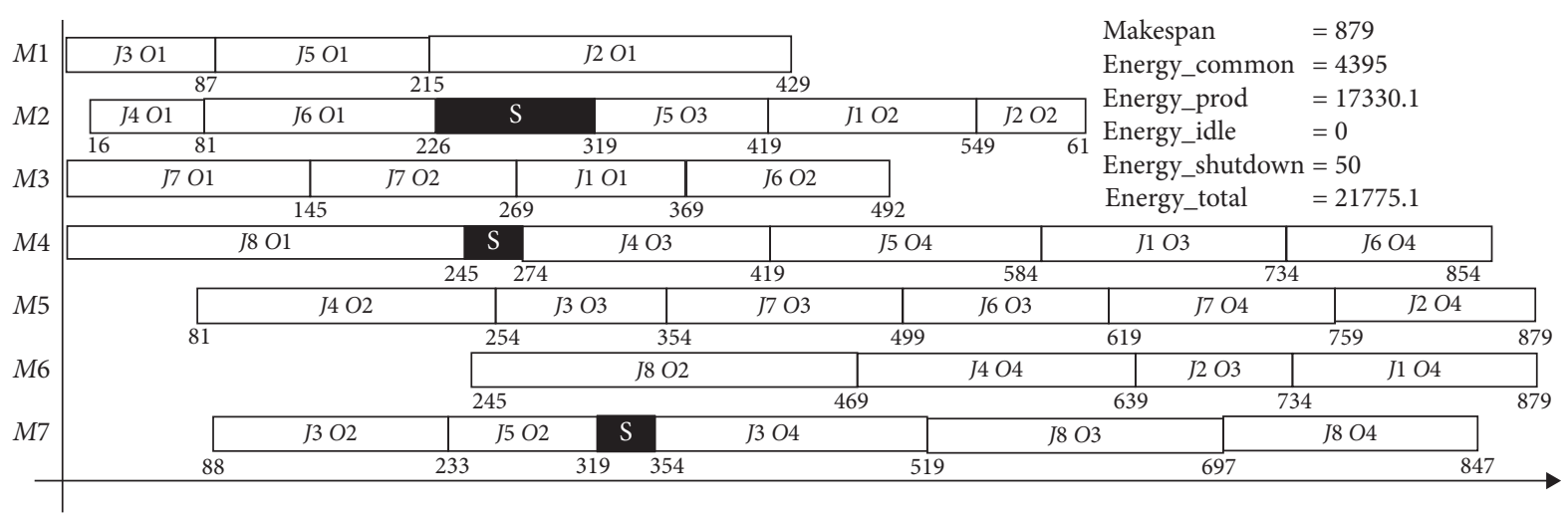

(b)

FIGURE 3: Schedules for the mfjs07 instance. (a) Traditional scheduling to minimize $C_{\max }$. (b) Energy-aware scheduling to minimize energy consumption while keeping the same productivity.

TABLE 2: Characteristics of the test instances.

\begin{tabular}{|c|c|c|c|c|c|}
\hline \multicolumn{4}{|c|}{ Fattahi instances } & \multicolumn{2}{|c|}{ Behnke instances } \\
\hline Name & Size & Name & Size & Name & Size \\
\hline sfjs01 & $2 / 2 / 2$ & mfjs01 & $5 / 3 / 6$ & Behnke1 & $10 / 3 / 20$ \\
\hline sfjs02 & $2 / 2 / 2$ & $\mathrm{mfjs} 02$ & $5 / 3 / 7$ & Behnke2 & $10 / 3 / 20$ \\
\hline sfjs 03 & $3 / 2 / 2$ & $\mathrm{mfjs} 03$ & $6 / 3 / 7$ & Behnke3 & $10 / 3 / 20$ \\
\hline sfjs04 & $3 / 2 / 2$ & $\mathrm{mfjs} 04$ & $7 / 3 / 7$ & Behnke4 & $10 / 3 / 20$ \\
\hline sfjs05 & $3 / 2 / 2$ & $\mathrm{mfjs} 05$ & $7 / 3 / 7$ & Behnke5 & $10 / 3 / 20$ \\
\hline sfjs06 & $3 / 3 / 2$ & mfjs06 & $8 / 3 / 7$ & Behnke6 & $20 / 3 / 20$ \\
\hline sfjs07 & $3 / 3 / 5$ & $\mathrm{mfjs} 07$ & $8 / 4 / 7$ & Behnke7 & $20 / 3 / 20$ \\
\hline sfjs08 & $3 / 3 / 4$ & $\mathrm{mfjs} 08$ & $9 / 4 / 8$ & Behnke8 & $20 / 3 / 20$ \\
\hline sfjs09 & $3 / 3 / 3$ & mfjs09 & $11 / 4 / 8$ & Behnke9 & $20 / 3 / 20$ \\
\hline sfjs 10 & $4 / 3 / 5$ & mfjs10 & $12 / 4 / 8$ & Behnke10 & $20 / 3 / 20$ \\
\hline \multicolumn{6}{|c|}{ Kacem instances } \\
\hline Name & Size & Name & Size & Name & Size \\
\hline Kacem 1 & $8 / 4 / 8$ & Kacem2 & $10 / 3 / 10$ & Kacem 3 & $15 / 4 / 10$ \\
\hline
\end{tabular}

MILP-3, and GA methods, while there is no statistically significant difference between MILP-2A and CP-2.

The previous tables compared the performance of models with a fixed computation time. It is necessary to check how the performance changes according to different computation times. Table 6 summarizes the total energy consumption in terms of computational run time for the Kacem's instances. Both CP and MILP models proved the optimality of Kacem1 instance within $60 \mathrm{~s}$. CP managed to prove the optimality of
Kacem 2 instance within 60 s, while MILP spent 150 s. In the Kacem 3 instance, the CP-2 found an optimal solution within $60 \mathrm{~s}$, while MILP-2A could not find an optimal solution within $600 \mathrm{~s}$. In addition, the CP-2 found a much better solution, in just 10 seconds, than the one MILP-2A found in 600 seconds. This experiment demonstrates that $\mathrm{CP}$ is quick to generate efficient (or optimal) solutions.

The energy saving does not appeal to highly capitalized manufacturing industries, such as automobiles and 


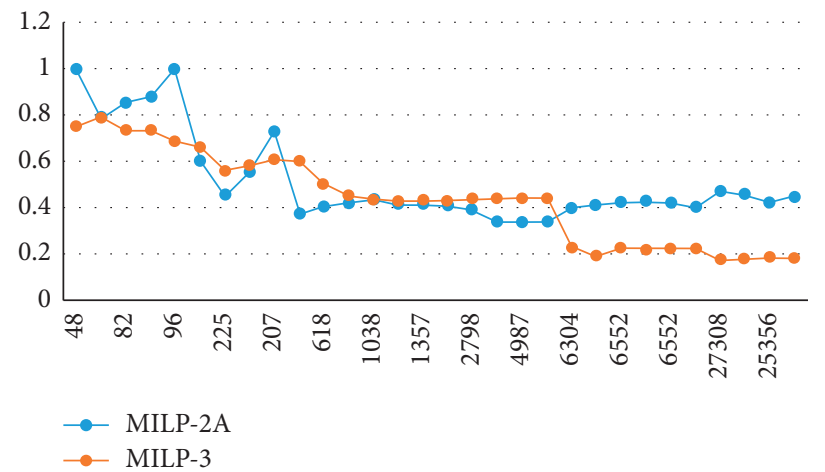

Figure 4: Comparison of the number of binary variables of MILP models.

TABle 3: Comparison of models in terms of total cost and computation times on Fattahi instances.

\begin{tabular}{|c|c|c|c|c|c|c|c|c|c|c|c|c|}
\hline \multirow{2}{*}{ Instance } & \multicolumn{6}{|c|}{ Total energy consumption } & \multicolumn{6}{|c|}{ Computation time (second) } \\
\hline & $\mathrm{CP}-1$ & $\mathrm{CP}-2$ & MILP-2 & MILP-2A & MILP-3 & GA & $\mathrm{CP}-1$ & $\mathrm{CP}-2$ & MILP-2 & MILP-2A & MILP-3 & GA \\
\hline sfjs01 & 815.2 & 815.2 & 815.2 & 815.2 & 815.2 & 815.2 & 54.6 & 0.3 & 0.0 & 0.0 & 0.0 & 0.2 \\
\hline sfjs02 & 1362.2 & 1362.2 & 1362.2 & 1362.2 & 1362.2 & 1362.2 & 65.4 & 0.3 & 0.0 & 0.0 & 0.0 & 0.2 \\
\hline sfjs03 & 2806.2 & 2806.2 & 2806.2 & 2806.2 & 2806.2 & 2806.2 & 507.3 & 0.3 & 0.0 & 0.0 & 0.0 & 0.2 \\
\hline sfjs04 & 4560.3 & 4560.3 & 4560.3 & 4560.3 & 4560.3 & 4560.3 & 600.1 & 0.3 & 0.0 & 0.0 & 0.0 & 0.2 \\
\hline sfjs05 & 1405.4 & 1405.4 & 1405.4 & 1405.4 & 1405.4 & 1405.4 & 547.7 & 0.3 & 0.0 & 0.0 & 0.0 & 0.2 \\
\hline sfjs06 & 4304.6 & 4304.6 & 4304.6 & 4304.6 & 4304.6 & 4420.6 & 600.1 & 0.3 & 0.1 & 0.0 & 0.0 & 0.3 \\
\hline sfjs07 & 5256.0 & 5256.0 & 5256.0 & 5256.0 & 5256.0 & 5345.2 & 600.0 & 0.3 & 0.1 & 0.0 & 0.0 & 0.3 \\
\hline sfjs08 & 3429.7 & 3429.7 & 3429.7 & 3429.7 & 3429.7 & 3477.7 & 600.0 & 0.3 & 0.3 & 0.1 & 0.0 & 0.3 \\
\hline sfjs09 & 2848.0 & 2848.0 & 2848.0 & 2848.0 & 2848.0 & 3023.2 & 600.2 & 0.4 & 0.1 & 0.1 & 0.1 & 0.3 \\
\hline sfjs10 & 8877.0 & 8877.0 & 8877.0 & 8877.0 & 8877.0 & 8877 & 600.0 & 0.4 & 0.1 & 0.0 & 0.0 & 0.4 \\
\hline mfjs01 & 9380.7 & 9380.7 & 9380.7 & 9380.7 & 9380.7 & 9981.2 & 600.0 & 600.1 & 2.9 & 0.8 & 1.6 & 0.5 \\
\hline $\mathrm{mfjs02}$ & 8642.0 & 8642.0 & 8642.0 & 8642.0 & 8642.0 & 9082 & 600.0 & 600.0 & 2.8 & 1.1 & 1.9 & 0.5 \\
\hline $\mathrm{mfjs03}$ & 10799.4 & 10762.8 & 10757.8 & 10757.8 & 10757.8 & 11700 & 600.0 & 600.0 & 35.3 & 4.7 & 10.8 & 0.6 \\
\hline mfjs04 & 13075.9 & 13038.6 & 13038.6 & 13038.6 & 13038.6 & 13696 & 600.0 & 600.1 & 117.5 & 27.3 & 82.3 & 0.6 \\
\hline mfjs05 & 12744.4 & 12600.1 & 12600.1 & 12600.1 & 12600.1 & 13579.1 & 600.0 & 600.1 & 93.3 & 23.7 & 426.8 & 0.6 \\
\hline mfjs06 & 15169.7 & 14960.1 & 14960.1 & 14960.1 & 14960.1 & 16257.5 & 600.0 & 600.1 & 49.6 & 16.7 & 52.0 & 0.7 \\
\hline mfjs07 & 20842.1 & 20542.1 & 21098.3 & 20990.3 & 21179.6 & 21884.9 & 600.0 & 600.0 & 607.5 & 600.2 & 600.3 & 1.0 \\
\hline mfjs08 & 24295.0 & 23763.8 & 24289.6 & 23999.0 & 23993.4 & 25812.3 & 600.0 & 600.1 & 601.8 & 600.3 & 600.3 & 1.0 \\
\hline mfjs09 & 30999.4 & 29788.1 & 31386.7 & 30882.5 & 30846.4 & 32237.1 & 600.1 & 600.0 & 601.1 & 600.5 & 600.1 & 1.2 \\
\hline $\mathrm{mfj}$ s10 & 34946.8 & 34410.7 & 36794.5 & 35168.6 & 37136.6 & 37293.6 & 600.0 & 600.1 & 600.4 & 601.6 & 600.8 & 1.5 \\
\hline Average & 10828.0 & 10677.7 & 10930.7 & 10804.2 & 10910.0 & 11380.8 & 538.8 & 300.2 & 135.7 & 123.9 & 148.9 & 0.5 \\
\hline
\end{tabular}

TABle 4: Comparison of models in terms of total cost and computation times on Behnke instances.

\begin{tabular}{|c|c|c|c|c|c|c|c|c|c|c|c|c|}
\hline \multirow{2}{*}{ Instance } & \multicolumn{6}{|c|}{ Total energy consumption } & \multicolumn{6}{|c|}{ Computation time (second) } \\
\hline & $\mathrm{CP}-1$ & $\mathrm{CP}-2$ & MILP-2 & MILP-2A & MILP-3 & GA & $\mathrm{CP}-1$ & $\mathrm{CP}-2$ & MILP-2 & MILP-2A & MILP-3 & GA \\
\hline Behnke1 & 1839.2 & 1795.8 & 1795.8 & 1795.8 & 1795.8 & 1889.3 & 600.0 & 600.1 & 600.1 & 104.6 & 600.1 & 1.1 \\
\hline Behnke2 & 1787.8 & 1768.0 & 1763.9 & 1763.9 & 1763.9 & 1884.6 & 600.0 & 600.1 & 318.3 & 167.3 & 180.8 & 1.1 \\
\hline Behnke3 & 1752.4 & 1749.9 & 1749.9 & 1749.9 & 1749.9 & 1902.6 & 600.0 & 600.2 & 332.6 & 55.3 & 309.2 & 1.1 \\
\hline Behnke4 & 2012.4 & 1964.2 & 1961.4 & 1945.9 & 1950.4 & 2082.9 & 600.0 & 600.1 & 600.2 & 379.7 & 600.1 & 1.1 \\
\hline Behnke5 & 1928.2 & 1865.7 & 1867.3 & 1865.7 & 1872.6 & 2101.1 & 600.0 & 600.1 & 600.3 & 124.4 & 600.1 & 1.0 \\
\hline Behnke6 & 3565.1 & 3354.1 & 3385.8 & 3361.3 & 3381.6 & 3637.7 & 600.1 & 600.2 & 600.4 & 600.2 & 600.1 & 2.2 \\
\hline Behnke7 & 3875.4 & 3483.1 & 3475.3 & 3454.6 & 3455.2 & 3734.9 & 600.0 & 600.2 & 602.7 & 600.3 & 600.3 & 2.2 \\
\hline Behnke8 & 3701.7 & 3445.2 & 3509.6 & 3474.4 & 3521.3 & 3774.9 & 600.0 & 600.3 & 604.4 & 600.4 & 600.3 & 1.9 \\
\hline Behnke9 & 3526.5 & 3326.6 & 3324.4 & 3294.4 & 3340.0 & 3579.2 & 600.0 & 600.3 & 600.4 & 600.3 & 600.2 & 2.1 \\
\hline Behnke10 & 4150.0 & 3703.4 & 3742.8 & 3705.7 & 3784.6 & 3916.8 & 600.0 & 600.3 & 603.3 & 600.4 & 600.3 & 2.1 \\
\hline Average & 2813.9 & 2645.6 & 2657.6 & 2641.2 & 2661.5 & 2850.4 & 600.0 & 600.2 & 546.3 & 383.3 & 529.1 & 1.6 \\
\hline
\end{tabular}

semiconductors, because the total energy cost is not even comparable to the revenue the manufacturers yield, so their sole goal is to maximize productivity. However, if we can maintain the same productivity, while significantly reducing energy consumption, the energy-aware production scheduling will be embraced by the industries. This type of 
TABLE 5: Comparison of models in terms of $t$-test: paired two-sample for means.

\begin{tabular}{lccccccccccc}
\hline \multirow{2}{*}{ CP-2 vs. } & \multicolumn{3}{c}{ Medium-sized Fattahi instances (MFJS01-10) } & \multirow{2}{*}{ MILP-2A vs. } & \multicolumn{4}{c}{ Behnke instances } \\
& CP-1 & MILP-2 & MILP-2A & MILP-3 & GA & & CP-1 & CP-2 & MILP-2 & MILP-3 & GA \\
\hline$t$-value $t(9)$ & -2.511 & -1.918 & -2.038 & -1.684 & -5.185 & $t$-value $t(9)$ & -3.387 & -0.780 & -3.426 & -2.337 & -8.547 \\
$p$-value & 0.0333 & 0.0874 & 0.0720 & 0.1265 & 0.0006 & $p$-value & 0.0080 & 0.4552 & 0.0076 & 0.0442 & 0.0000 \\
\hline
\end{tabular}

TABLE 6: Comparison of models in terms of total energy consumption according to different CPU times.

\begin{tabular}{|c|c|c|c|c|c|c|c|c|c|c|}
\hline \multirow{2}{*}{ Instance } & \multirow{2}{*}{ Model } & \multicolumn{9}{|c|}{$\mathrm{CPU}(\mathrm{sec})$} \\
\hline & & 10 & 30 & 60 & 90 & 120 & 150 & 180 & 300 & 600 \\
\hline \multirow{3}{*}{ Kacem1 } & CP-2 & 407.5 & 401.4 & 393.4 & - & - & - & - & - & - \\
\hline & MILP-2A & 443.0 & 408.6 & 393.4 & - & - & - & - & - & - \\
\hline & GA & 422.6 & & & & & & & & \\
\hline \multirow[b]{2}{*}{ Kacem2 } & CP-2 & 205.8 & 202.2 & 200.8 & - & - & - & - & - & - \\
\hline & $\begin{array}{c}\text { MILP-2A } \\
\text { GA }\end{array}$ & $\begin{array}{l}238.6 \\
227.5\end{array}$ & 220.8 & 215.5 & 210.0 & 201.5 & 200.8 & - & - & - \\
\hline \multirow[b]{2}{*}{ Kacem3 } & CP-2 & 520.2 & 435.8 & 435.8 & 435.8 & 435.8 & 435.8 & 435.8 & 435.8 & 435.8 \\
\hline & $\begin{array}{c}\text { MILP-2A } \\
\text { GA }\end{array}$ & $\begin{array}{c}1909.2 \\
501.8\end{array}$ & 1908.1 & 1908.1 & 1908.1 & 1908.1 & 1908.1 & 1908.1 & 1908.1 & 1908.1 \\
\hline
\end{tabular}

TABle 7: Potential energy saving based on Fattahi instances.

\begin{tabular}{|c|c|c|c|c|c|}
\hline \multirow{3}{*}{ Instance } & \multicolumn{3}{|c|}{ Total energy consumption } & \multirow{2}{*}{\multicolumn{2}{|c|}{$\begin{array}{c}\text { Energy saving } \\
\text { Energy-aware scheduling }\end{array}$}} \\
\hline & \multirow{2}{*}{ Tradition } & \multicolumn{2}{|c|}{ Energy-aware scheduling } & & \\
\hline & & w/o shutdown & w/ shutdown & w/o shutdown (\%) & w/ shutdown (\%) \\
\hline sfjs01 & 815.2 & 815.2 & 815.2 & 0.00 & 0.00 \\
\hline sfjs02 & 1362.2 & 1362.2 & 1362.2 & 0.00 & 0.00 \\
\hline sfjs03 & 2806.2 & 2806.2 & 2806.2 & 0.00 & 0.00 \\
\hline sfjs04 & 4560.3 & 4560.3 & 4560.3 & 0.00 & 0.00 \\
\hline sfjs05 & 1405.4 & 1405.4 & 1405.4 & 0.00 & 0.00 \\
\hline sfjs06 & 4630.6 & 4360.6 & 4360.6 & 5.83 & 5.83 \\
\hline sfjs07 & 5530.2 & 5304.2 & 5304.2 & 4.09 & 4.09 \\
\hline sfjs08 & 3599.2 & 3599.2 & 3599.2 & 0.00 & 0.00 \\
\hline sfjs09 & 3121.0 & 2951.0 & 2951.0 & 5.45 & 5.45 \\
\hline sfjs10 & 9217.8 & 8893.0 & 8877.0 & 3.52 & 3.70 \\
\hline mfjs01 & 9498.6 & 9468.6 & 9468.6 & 0.32 & 0.32 \\
\hline mfjs02 & 9036.2 & 8918.2 & 8918.2 & 1.31 & 1.31 \\
\hline mfjs03 & 11410.0 & 11356.0 & 11278.0 & 0.47 & 1.16 \\
\hline mfjs04 & 13233.9 & 13163.9 & 13075.9 & 0.53 & 1.19 \\
\hline mfjs05 & 13333.6 & 13293.6 & 13293.6 & 0.30 & 0.30 \\
\hline mfjs06 & 16443.1 & 16086.7 & 16086.7 & 2.17 & 2.17 \\
\hline mfjs07 & 22072.9 & 21973.1 & 21775.1 & 0.45 & 1.35 \\
\hline mfjs08 & 24884.4 & 24509.6 & 24503.8 & 1.51 & 1.53 \\
\hline mfjs09 & 32144.4 & 31262.2 & 31262.2 & 2.74 & 2.74 \\
\hline mfjs10 & 35857.7 & 34874.9 & 34874.9 & 2.74 & 2.74 \\
\hline & & rage & & 1.57 & 1.69 \\
\hline
\end{tabular}

mathematical approach is known as epsilon-constraint method, where one of the objectives is taken as a single objective function and the others are included into a model as constraint [51].

Tables 7 and 8 attempt to meet this demand by comparing the traditional scheduling and energy-aware scheduling. The traditional scheduling minimizes the makespan (cycle time reduction), whereas the energy-aware scheduling minimizes the total energy consumption as it maintains the same makespan. Column 1 identifies the name of the instance. Column 2 reports the total energy consumption when the model solves each test instance with the makespan minimization. Columns 3-4 report the total energy consumption when the model runs with the energy consumption minimization with the same makespan. This experimentation identifies potential energy-saving without compromising productivity. For Fattahi's instances, Table 7 shows $1.57 \%$ and $1.69 \%$ saving without and with shutdown 
TABle 8: Potential energy saving based on Behnke instances.

\begin{tabular}{|c|c|c|c|c|c|}
\hline \multirow{3}{*}{ Instance } & \multicolumn{3}{|c|}{ Total energy consumption } & \multirow{2}{*}{\multicolumn{2}{|c|}{$\begin{array}{c}\text { Energy saving } \\
\text { Instance }\end{array}$}} \\
\hline & \multirow{2}{*}{ Tradition } & \multicolumn{2}{|c|}{ Energy-aware scheduling } & & \\
\hline & & w/o shutdown & w/ shutdown & w/o shutdown (\%) & w/ shutdown (\%) \\
\hline Behnke1 & 2156.2 & 1809.9 & 1809.9 & 16.06 & 16.06 \\
\hline Behnke2 & 1955.2 & 1789.3 & 1789.3 & 8.49 & 8.49 \\
\hline Behnke3 & 2106.1 & 1840.9 & 1796.3 & 12.59 & 14.71 \\
\hline Behnke4 & 2237.8 & 1945.9 & 1945.9 & 13.04 & 13.04 \\
\hline Behnke5 & 2125.1 & 1953.2 & 1953.2 & 8.09 & 8.09 \\
\hline Behnke6 & 4356.7 & 3358.3 & 3358.3 & 22.92 & 22.92 \\
\hline Behnke7 & 4210.8 & 3543.7 & 3543.7 & 15.84 & 15.84 \\
\hline Behnke8 & 4200.7 & 3502.8 & 3502.8 & 16.61 & 16.61 \\
\hline Behnke9 & 4022.5 & 3341.8 & 3297.9 & 16.92 & 18.01 \\
\hline Behnke10 & 4558.5 & 3740.1 & 3714.5 & 17.95 & 18.51 \\
\hline \multicolumn{4}{|c|}{ Average } & 14.85 & 15.23 \\
\hline
\end{tabular}

TABLE 9: Energy saving according to different energy consumption.

\begin{tabular}{lcccc}
\hline Instance & $E_{o, k}(\%)$ & $2 E_{o, k}(\%)$ & $3 E_{o, k}(\%)$ & $4 E_{o, k}(\%)$ \\
\hline Kacem1 & 10.5 & 9.5 & 9.2 & 8.9 \\
Kacem2 & 2.8 & 1.7 & 1.3 & 1.1 \\
Kacem3 & 11.4 & 9.7 & 8.9 & 8.6 \\
Average & 8.2 & 7.0 & 6.5 & 6.2 \\
\hline
\end{tabular}

strategy, respectively. The mild saving can be explained by the test instance itself.

Table 8 reports the results of Behnke's instances. This experimentation identified the potential energy-saving without compromising productivity by $14.85 \%$ (w/o shutdown) and $15.23 \%$ (w/shutdown). The increased savings compared with the ones with Fattahi's instance are due to the size of this Behnke's instance, allowing the solver the flexibility to find better solutions. In order to determine if the means of two sets of data are significantly different from each other, we conducted the $t$-test: Paired Two-Sample for Means at an alpha level of 0.05 . The test indicates that there is a statistically significant difference between traditional and energy-aware methods with $t(19)=3.5$ and $p<0.01$ in Fattahi's instance and $t(9)=5.53$ and $p<0.001$ in Behnke's instance.

Table 9 records the amount of energy saving by applying the proposed energy-aware method according to different multipliers on $E_{o, k}$. This experimentation identified the potential energy-saving without compromising productivity by $6-8 \%$. The improvement was slightly decreased as the multiplier was increased.

\section{Conclusion}

We have investigated the energy-efficient FJSP with a shutdown (on/off) strategy to save idle energy consumption. The shutdown can be enforced when a continuous idle period of a machine is expected to be long enough to compensate for the shutdown penalty. An alternative MILP model is proposed. Then, a novel constraint programming is proposed. Finally, practical operational scenarios are examined.
The computational study demonstrated that (1) the proposed models significantly outperform the best benchmark model (MILP-2) and (2) we can maintain the maximum productivity while significantly reducing the energyconsumption by $14.85 \%$ (w/o shutdown) and $15.23 \%$ (w/ shutdown) on average, thus promoting energy-aware production scheduling to highly capitalized manufacturing industries. We offer benchmarking instances, CPLEX MIP, $\mathrm{CP}$, and GA source codes, which have been used in this research, in order to promote related research, thus expediting the adoption of energy-efficient scheduling in manufacturing facilities.

In future research, we will extend the proposed models to consider time-of-use (TOU) electricity and peak power load since the energy cost can be further saved. Utility companies across the U.S. are offering TOU-based electricity demand response programs. The key is to shift productions to offpeak periods.

\section{Data Availability}

The data used to support the findings of this study have been deposited in the GitHub repository (https://github.com/ hamcruise/FJSP-Shutdown)

\section{Conflicts of Interest}

The authors declare that there are no conflicts of interest regarding the publication of this article.

\section{Acknowledgments}

This work was supported by the National Research Foundation of Korea (NRF) grant funded by the Korean Government (MSIT) (no. 2019R1G1A1085191).

\section{References}

[1] "International energy outlook 2019 with projections to 2050," 2019, https://www.eia.gov/ieo.

[2] International Energy Agency, Tracking Industrial Energy Efficiency and CO2 Emissions, Organisation for Economic Co-Operation and Development, Paris, France, 2007. 
[3] G. May, B. Stahl, M. Taisch, and V. Prabhu, "Multi-objective genetic algorithm for energy-efficient job shop scheduling," International Journal of Production Research, vol. 53, no. 23, pp. 7071-7089, 2015.

[4] A. M. Stark, U.S. Energy Use Rises to Highest Level Ever, Lawrence Livermore National Laboratory (LLNL) News Release, Livermore, CA, USA, 2019.

[5] G. Mouzon, M. B. Yildirim, and J. Twomey, "Operational methods for minimization of energy consumption of manufacturing equipment," International Journal of Production Research, vol. 45, no. 18-19, pp. 4247-4271, 2007.

[6] Y. He, F. Liu, H.-J. Cao, and C.-B. Li, "A bi-objective model for job-shop scheduling problem to minimize both energy consumption and makespan," Journal of Central South University of Technology, vol. 12, no. 2, pp. 167-171, 2005.

[7] A. Che, X. Wu, J. Peng et al., "Energy-efficient bi-objective single-machine scheduling with power-down mechanism," Computers \& Opereations Research, vol. 85, pp. 172-183, 2017.

[8] L. Zhang, Q. Tang, Z. Wu, and F. Wang, "Mathematical modeling and evolutionary generation of rule sets for energyefficient flexible job shops," Energy, vol. 138, pp. 210-227, 2017.

[9] L. Meng, C. Zhang, X. Shao, and Y. Ren, "MILP models for energy-aware flexible job shop scheduling problem," Journal of Cleaner Production, vol. 210, pp. 710-723, 2019.

[10] I. A. Chaudhry and A. A. Khan, "A research survey: review of flexible job shop scheduling techniques," International Transactions in Operational Research, vol. 23, no. 3, pp. 551-591, 2016.

[11] H. M. Wagner, "An integer linear-programming model for machine scheduling," Naval Research Logistics Quarterly, vol. 6, no. 2, pp. 131-140, 1959.

[12] P. Fattahi, M. Saidi Mehrabad, and F. Jolai, "Mathematical modeling and heuristic approaches to flexible job shop scheduling problems," Journal of Intelligent Manufacturing, vol. 18, no. 3, pp. 331-342, 2007.

[13] N. Imanipour, "Modeling \& solving flexible job shop problem with sequence dependent setup times," in Proceedings of the International Conference on Service Systems and Service Management, vol. 2, pp. 1205-1210, Troyes, France, October 2006.

[14] C. Özgüven, L. Özbakır, and Y. Yavuz, "Mathematical models for job-shop scheduling problems with routing and process plan flexibility," Applied Mathematical Modelling, vol. 34, no. 6, pp. 1539-1548, 2010.

[15] I.-C. Choi and D.-S. Choi, "A local search algorithm for jobshop scheduling problems with alternative operations and sequence-dependent setups," Computers \& Industrial Engineering, vol. 42, no. 1, pp. 43-58, 2002.

[16] M. C. Gomes, A. P. Barbosa-Póvoa, and A. Q. Novais, "Optimal scheduling for flexible job shop operation," International Journal of Production Research, vol. 43, no. 11, pp. 2323-2353, 2005.

[17] Y. Demir and S. Kürşat İşleyen, "Evaluation of mathematical models for flexible job-shop scheduling problems," Applied Mathematical Modelling, vol. 37, no. 3, pp. 977-988, 2013.

[18] Q. Jiang, X. Liao, R. Zhang, and Q. Lin, "Energy-saving production scheduling in a single-machine manufacturing system by improved particle swarm optimization," Mathematical Problems in Engineering, vol. 2020, Article ID 8870917, 16 pages, 2020.

[19] J.-Q. Li, H.-Y. Sang, Y.-Y. Han, C.-G. Wang, and K.-Z. Gao, "Efficient multi-objective optimization algorithm for hybrid flow shop scheduling problems with setup energy consumptions," Journal of Cleaner Production, vol. 181, pp. 584-598, 2018.

[20] B. Zhang, Q. K. Pan, L. Gao, L. L. Meng, X. Y. Li, and K. K. Peng, "A three-stage multiobjective approach based on decomposition for an energy-efficient hybrid flow shop scheduling problem," IEEE Transactions on Systems, Man, and Cybernetics: Systems, vol. 50, no. 12, pp. 4984-4999, 2019.

[21] T. Jiang, C. Zhang, H. Zhu, and G. Deng, "Energy-efficient scheduling for a job shop using grey wolf optimization algorithm with double-searching mode," Mathematical Problems in Engineering, vol. 2018, Article ID 8574892, 12 pages, 2018.

[22] L. Yin, X. Li, L. Gao, C. Lu, and Z. Zhang, "A novel mathematical model and multi-objective method for the lowcarbon flexible job shop scheduling problem," Sustainable Computing: Informatics and Systems, vol. 13, pp. 15-30, 2017.

[23] Z. Jiang, L. Zuo, and E. Mingcheng, "Study on multi-objective flexible job-shop scheduling problem considering energy consumption," Journal of Industrial and Engineering and Management, vol. 7, no. 3, pp. 589-604, 2014.

[24] L. Meng, C. Zhang, X. Shao, Y. Ren, and C. Ren, "Mathematical modelling and optimisation of energy-conscious hybrid flow shop scheduling problem with unrelated parallel machines," International Journal of Production Research, vol. 57, no. 4, pp. 1119-1145, 2019.

[25] M. Dai, D. Tang, A. Giret, M. A. Salido, and W. D. Li, "Energyefficient scheduling for a flexible flow shop using an improved genetic-simulated annealing algorithm," Robotics and Computer-Integrated Manufacturing, vol. 29, no. 5, pp. 418-429, 2013.

[26] X. Wu and Y. Sun, "A green scheduling algorithm for flexible job shop with energy-saving measures," Journal of Cleaner Production, vol. 172, pp. 3249-3264, 2018.

[27] L. Meng, C. Zhang, B. Zhang, and Y. Ren, "Mathematical modeling and optimization of energy-conscious flexible job shop scheduling problem with worker flexibility," IEEE Access, vol. 7, pp. 68043-68059, 2019.

[28] A. Che, K. Lv, E. Levner, and V. Kats, "Energy consumption minimization for single machine scheduling with bounded maximum tardiness," in Proceedings of the ICNSC 2015-2015 IEEE 12th International Conference of Networking, pp. 146150, Taipei, Taiwan, April 2015.

[29] K. Fang, N. A. Uhan, F. Zhao, and J. W. Sutherland, "Flow shop scheduling with peak power consumption constraints," Annals of Operations Research, vol. 206, no. 1, pp. 115-145, 2013.

[30] S. A. Mansouri, E. Aktas, and U. Besikci, "Green scheduling of a two-machine flowshop: trade-off between makespan and energy consumption," European Journal of Operational Research, vol. 248, no. 3, pp. 772-788, 2016.

[31] R. Zhang and R. Chiong, "Solving the energy-efficient job shop scheduling problem: a multi-objective genetic algorithm with enhanced local search for minimizing the total weighted tardiness and total energy consumption," Journal of Cleaner Production, vol. 112, pp. 3361-3375, 2016.

[32] L. Zhang, X. Li, L. Gao, G. Zhang, and X. Wen, "Dynamic scheduling model in FMS by considering energy consumption and schedule efficiency," in Proceedings of the IEEE International Conference on Computer Supported Cooperative Work in Design, Wuhan, China, May 2012.

[33] M. Rager, C. Gahm, and F. Denz, "Energy-oriented scheduling based on evolutionary algorithms," Computers \& Operations Research, vol. 54, pp. 218-231, 2015. 
[34] S. Schulz, J. S. Neufeld, and U. Buscher, "A multi-objective iterated local search algorithm for comprehensive energyaware hybrid flow shop scheduling," Journal of Cleaner Production, vol. 224, pp. 421-434, 2019.

[35] J.-Y. Moon and J. Park, "Smart production scheduling with time-dependent and machine-dependent electricity cost by considering distributed energy resources and energy storage," International Journal of Production Research, vol. 52, no. 13, pp. 3922-3939, 2014.

[36] X. Gong, T. De Pessemier, L. Martens, and W. Joseph, "Energy- and labor-aware flexible job shop scheduling under dynamic electricity pricing: a many-objective optimization investigation," Journal of Cleaner Production, vol. 209, pp. 1078-1094, 2019.

[37] C. Gahm, F. Denz, M. Dirr, and A. Tuma, "Energy-efficient scheduling in manufacturing companies: a review and research framework," European Journal of Operational Research, vol. 248, no. 3, pp. 744-757, 2016.

[38] K. Gao, Y. Huang, A. Sadollah, and L. Wang, "A review of energy-efficient scheduling in intelligent production systems," Complex \& Intelligent Systems, vol. 6, pp. 1-13, 2019.

[39] F. S. Hiller and G. J. Lieberman, Introduction to Operations Research, Prentice Hall, Hoboken, NJ, USA, 10th edition, 2015.

[40] P. Laborie, J. Rogerie, P. Shaw, and P. Vilím, "IBM ILOG CP optimizer for scheduling," Constraints, vol. 23, no. 2, pp. 210-250, 2018.

[41] A. Ham, "Transfer-robot task scheduling in job shop," International Journal of Production Research, vol. 59, no. 3, pp. 813-823, 2020.

[42] IBM Software, IBM ILOG CPLEX Optimization Studio V12.8.0, IBM, Endicott, NY, USA, 2019.

[43] D. Behnke and M. J. Geiger, Test Instances For The Flexible Job Shop Scheduling Problem With Work Centers, HelmutSchmidt-Universität, Hamburg, Germany, 2012.

[44] I. Kacem, S. Hammadi, and P. Borne, "Approach by localization and multiobjective evolutionary optimization for flexible job-shop scheduling problems," IEEE Transactions on Systems, Man and Cybernetics, Part C (Applications and Reviews), vol. 32, no. 1, pp. 1-13, 2002.

[45] M. R. Singh and S. S. Mahapatra, "A quantum behaved particle swarm optimization for flexible job shop scheduling," Computers \& Industrial Engineering, vol. 93, pp. 36-44, 2016.

[46] J. Gao, L. Sun, and M. Gen, "A hybrid genetic and variable neighborhood descent algorithm for flexible job shop scheduling problems," Computers \& Operations Research, vol. 35, no. 9, pp. 2892-2907, 2008.

[47] K. Gao, Z. Cao, L. Zhang, Z. Chen, Y. Han, and Q. Pan, “A review on swarm intelligence and evolutionary algorithms for solving flexible job shop scheduling problems," IEEE/CAA Journal of Automatica Sinica, vol. 6, no. 4, pp. 904-916, 2019.

[48] M. A. González, A. Oddi, and R. Rasconi, "Efficient approaches for solving a multiobjective energy-aware job shop scheduling problem," Fundamenta Informaticae, vol. 167, no. 1-2, pp. 93-132, 2019.

[49] F. Pezzella, G. Morganti, and G. Ciaschetti, "A genetic algorithm for the flexible job-shop scheduling problem," Computers \& Operations Research, vol. 35, no. 10, pp. 32023212, 2008.

[50] G. Zhang, L. Gao, and Y. Shi, "An effective genetic algorithm for the flexible job-shop scheduling problem," Expert Systems with Applications, vol. 38, no. 4, pp. 3563-3573, 2011.
[51] H. G. Resat, "Design and analysis of novel hybrid multiobjective optimization approach for data-driven sustainable delivery systems," IEEE Access, vol. 8, pp. 90280-90293, 2020. 